\title{
Exercício físico e surdez: Uma revisão integrativa
}

\author{
Physical exercise and deafness: A integrative review \\ Ejercicio físico y sordera: Una revisión integradora
}

Recebido: 29/06/2021 | Revisado: 06/07/2021 | Aceito: 08/07/2021 | Publicado: 19/07/2021

\author{
Ananda Liliane Oliveira Santana \\ ORCID: https://orcid.org/0000-0001-5825-0123 \\ Centro Universitário Santo Agostinho, Brasil \\ E-mail: anandasantanaa18@gmail.com \\ Guilherme Soares Portela Lobo \\ ORCID: https://orcid.org/0000-0002-0909-9639 \\ Centro Universitário Santo Agostinho, Brasil \\ E-mail: guipmartins@hotmail.com \\ Kácio dos Santos Silva \\ ORCID: https://orcid.org/0000-0003-1577-0270 \\ Centro Universitário Santo Agostinho, Brasil \\ E-mail: kaiosam@hotmail.com
}

\begin{abstract}
Resumo
A inclusão social dos surdos é um complexo fenômeno que engloba questões de cunho moral, conceitual, legal, entre outros. Neste caminho, a principal conquista dos surdos foi, sem dúvida, a oficialização da sua língua como língua oficial brasileira, que é fator de identificação, cultura e socialização para os surdos. Já o esporte teve vultoso papel no processo de organização e de transformação subjetiva dos surdos, o que, no contexto da Educação Ambiental, o caracteriza como ferramenta de modificação dos ambientes físicos e psicossociais por onde os surdos transitam, à medida que promove a autoestima dos indivíduos, a autonomia dos grupos e o reconhecimento dos demais. Sendo o esporte uma ferramenta de inserção social, os surdos percebem naturalmente que houve grande contribuição das práticas esportivas no processo de organização e de transformação subjetiva destes indivíduos. Neste contexto, a Confederação Brasileira de Desportos de Surdos (CBDS) é uma instituição de incomensurável importância para os surdos no Brasil, porém sua história não está registrada oficialmente, nem é completamente conhecida. Por isto, este estudo foi uma revisão integrativa, explorativa do tipo quantitativa e seletiva. Utilizou-se as bases de dados Capes, Scielo e google acadêmico. Analisou-se a relação do exercício físico e a surdez, quais as limitações do surdo frente a atividades físicas, quais as dificuldades encontradas com a inclusão do surdo nas práticas de exercício. Na pratica ainda existe muitas dificuldades de inclusão, levando em conta a falta de conhecimento da língua de sinais dos profissionais que trabalham diretamente com os surdos. Ainda existe muitos obstáculos a serem enfrentados, pois a inclusão possui barreiras, e que só será possível através de uma conscientização da população.
\end{abstract}

Palavras-chave: Surdez; Inclusão social; Exercício físico; Educação física; Treinamento.

\begin{abstract}
The social inclusion of the deaf is a complex phenomenon that encompasses questions of a moral, conceptual, legal nature, among others. In this way, the main achievement of the deaf was, without a doubt, the officialization of their language as an official Brazilian language, which is a factor of identification, culture and socialization for the deaf. Sport, on the other hand, played a major role in the process of organization and subjective transformation of the deaf, which, in the context of Environmental Education, characterizes it as a tool for modifying the physical and psychosocial environments in which the deaf transit, as it promotes the self-esteem of the deaf. individuals, the autonomy of the groups and the recognition of the others. As sport is a tool for social inclusion, deaf people naturally perceive that there was a great contribution from sports practices in the process of organization and subjective transformation of these individuals. In this context, the Brazilian Confederation of Sports for the Deaf (CBDS) is an institution of immeasurable importance for the deaf in Brazil, but its history is not officially registered, nor is it fully known. For this reason, this study was an integrative, exploratory review of the quantitative and selective type. The Capes, Scielo and google academic databases were used. The relationship between physical exercise and deafness was analyzed, which are the limitations of the deaf in relation to physical activities, which are the difficulties encountered with the inclusion of the deaf in the exercise practices. In practice, there are still many inclusion difficulties, taking into account the lack knowledge of the sign language of professionals who work directly with the deaf. There are still many obstacles to be faced, as inclusion has barriers, and that will only be possible through an awareness of the population.
\end{abstract}

Keywords: Deafness; Social inclusion; Exercise; Physical education; Training.

\section{Resumen}

La inclusión social de las personas sordas es un fenómeno complejo que engloba cuestiones de carácter moral, conceptual y legal, entre otras. De esta manera, el principal logro de los sordos fue, sin duda, la oficialización de su 
idioma como lengua oficial brasileña, que es un factor de identificación, cultura y socialización para los sordos. El deporte, por su parte, tuvo un papel importante en el proceso de organización y transformación subjetiva de las personas sordas, lo que, en el contexto de la Educación Ambiental, lo caracteriza como una herramienta para modificar los entornos físicos y psicosociales por los que se mueven las personas sordas. ya que promueve la autoestima de las personas, la autonomía de los grupos y el reconocimiento de los demás. Dado que el deporte es una herramienta de inclusión social, las personas sordas perciben naturalmente que hubo una gran contribución de las prácticas deportivas en el proceso de organización y transformación subjetiva de estos individuos. En este contexto, la Confederación Brasileña de Deportes para Sordos (CBDS) es una institución de inconmensurable importancia para los sordos en Brasil, pero su historia no está registrada oficialmente, ni es del todo conocida. Por tanto, este estudio fue una revisión integradora, exploratoria, cuantitativa y selectiva. Se utilizaron bases de datos de Capes, Scielo y Google académico. Se analizó la relación entre el ejercicio físico y la sordera, así como las limitaciones de los sordos en la actividad física y las dificultades encontradas con la inclusión de los sordos en las prácticas de ejercicio. En la práctica, aún existen muchas dificultades de inclusión, teniendo en cuenta el desconocimiento de la lengua de signos de los profesionales que trabajan directamente con sordos. Aún quedan muchos obstáculos por enfrentar, ya que la inclusión tiene barreras, y solo será posible a través de una conciencia de la población.

Palabras clave: Sordera; Inclusión social; Ejercicio Físico; Educación; Entrenamiento físico.

\section{Introdução}

A surdez é o déficit sensorial mais comum em todo o mundo. Afeta cerca de $5 \%$ da população mundial, atinge pessoas de todas as idades e tem um custo pessoal e social significativo, os países economicamente desenvolvidos apresentam taxas gerais mais baixas de perda auditiva em comparação com os países em desenvolvimento (Sheffield; Smith, 2019).

É comum o baixo nível de aptidão aeróbica em pessoas com deficiência auditiva o que pode ser explicado pela falta de aquisição de habilidades verbais que tenham um efeito positivo no desenvolvimento dos pulmões devido ao uso dos pulmões para vocalização (Akınoğlu; Kocahan, 2019; Tzanetakos et al., 2017). Nessa perspectiva a atividade física pode ser fundamental na vida desses indivíduos.

O esporte surdo é um artefato cultural de extrema importância para a comunidade surda. A organização dos surdos em atividades esportivas tem registros, que datam do século XX como o primeiro clube de futebol surdo do mundo, o Glasgow DF no Reino Unido, a associação Sports Club for the Deaf de 1888, em Berlim e o primeiro clube desportivo para surdos na França, o Club Cycliste des sourds-muets de 1899. Posteriormente, no início do século XX, muitas outras associações desportivas foram criadas ao redor do mundo (Franco, Palludo, Lebedeff, 2015).

No Brasil, de acordo com Monteiro (2006), as associações desportivas tiveram sua origem no Grêmio Esportivo fundado em 1930, no Instituto Nacional de Educação de Surdos (INES). O INES, que data de 1856, na década de 30, foi a única escola de surdos no país a congregar um número muito expressivo de surdos vindos de diversas partes do Brasil. O Grêmio Esportivo do INES foi responsável por elaborar ou adaptar regras de esportes e desportos e organizar competições internas em que a maioria, se não todos dos alunos, participavam com grande interesse. Estes eram importantes eventos de confraternização e interação entre surdos que promoviam a integração social daqueles indivíduos. Ocorre que, ao concluírem seus estudos no INES e retornarem à sua terra de origem, muitos surdos sentiam falta dos eventos esportivos e sociais promovidos pelo Grêmio Esportivo e sentiam-se impelidos pelo desejo de retomar e ampliar as possibilidades de convivência entre surdos, então fundaram várias associações de surdos Brasil afora.

A atividade física traz muitos benefícios para a saúde e a qualidade de vida da população, incluindo todas as pessoas gêneros e idades. Na adolescência existem muitas evidências que mostram que atividade física traz muitos benefícios relacionados à saúde esquelética e no controle da pressão sanguínea e da obesidade. Mesmo com tantos benefícios relacionados a prática a atividade física como promoção de saúde e preventivo contra doenças, a um grande déficit de pessoas que pratiquem algum tipo de atividade física.

Conforme Boaventura et al. (2009, p.43): “A atividade física proporciona a pessoa com deficiência, efeitos positivos, tanto no bem físico como no mental, a prática da atividade física, aumentam a motivação e a integração dessas pessoas com deficiência a estar preocupadas com sua saúde e que proporcionando hábitos saudáveis”. 
A surdez não acarreta nenhuma limitação motora e não interfere na prática de atividade física, sendo a prática, um mecanismo de trabalhar a consciência corporal e desenvolvimento das atividades motoras. Esse benefício também é encontrado em pessoas ouvintes, portanto de uma forma geral a surdez não interfere na parte motora do indivíduo. (Santos, 2011). Logo, uma pessoa com surdez consegue praticar atividade física sem ter nenhuma interferência motora, além disso, uma pessoa com surdez aguça outros sentidos, sua visão pode capitar grandes ações que um indivíduo ouvinte pode ou não ter a capacidade de perceber (Santos, 2011).

Nesse sentido, a democratização e universalização das oportunidades devem ser pensadas de maneira real, de acordo com a realidade, sem atos paternalistas ou protecionismos, mas buscando de fato, o respeito pelas diferenças. Não significa facilitar as condições de acesso à educação, lazer, moradia e saúde para os deficientes, mas na realidade, trata-se de oferecer condições ideais de igualdade. Pensar nesse panorama é acreditar na capacidade potencial de aprender e produzir conhecimentos, de pessoas com formas diferentes de se relacionar com o mundo e com o saber; é conhecer, concretamente, que existe o princípio da universalização (Basso; Capellini, 2012).

Conforme o exposto sobre exercício físico e surdez, devido a baixíssimos estudos abordando a temática e necessidade de discussão do tema, o presente estudo visa investigar através de uma revisão de integrativa a o contexto de exercício físico e surdez.

\section{Metodologia}

Trata-se de uma revisão integrativa de literatura, um método rígido e de natureza ampla, que visa identificar o conhecimento produzido sobre determinado tema. Além disso, fornece informações suficientes sobre o tema pesquisado, direcionando para a definição de conceitos, revisão de teorias ou análise metodológica (Ercole; Melo; Alcoforado, 2014), resultando em contribuições significativas.

O trabalho foi desenvolvido através de revisão integrativa, seguiu os preceitos dos estudos exploratórios, do tipo quantitativo e seletivos, baseado em materiais já elaborados, como artigos científicos, revistas e periódicos. A busca foi realizada através de descritores provenientes do DeCS (Descritores em Ciências da Saúde) e MeSH (Medical Subject Headings): "Exercício físico"; "Surdez"; "Deafness"; "Inclusão"; "Exercise"; "Social Inclusion”; "Inclusão Social”; "Perda Auditiva"; "Hearing Loss".

A busca dos artigos para obtenção dos resultados foi realizada na Biblioteca Virtual google academico incluindo os artigos indexados na base de dados: Base de Dados do Web of Science, no Scopus, nos portais de periódicos CAPES e Scientific Eletronic Library Online (SciELO).

Foram incluídos estudos publicados em português no período de 2008 a 2019 em periódicos nacionais e internacionais que ofereceram informações sobre o exercício físico e surdez, manuais, resumos de anais, revisões de literatura. Foram excluídos todos os artigos fora do recorte do temporal, além de livros, estudos duplicados e artigos fora da temática abordada.

A partir disto, diferentes artigos foram selecionados. Na sequência, foi desenvolvido resultados e discussão a respeito do tema abordado, correlacionando os achados de diferentes autores, para se alcançarem os objetivos aqui propostos.

\section{Resultados e Discussão}

Para a realização da pesquisa foi realizado uma pesquisa integrativa, seguindo os preceitos do estudo exploratório e seletivo, dos quais foram selecionados artigos, publicados nos últimos 11 anos (2008 a 2019). Durante a busca foram encontradas 3.550 publicações. Os artigos foram encontrados no Google Acadêmico, utilizando as bases de dados Capes e Scielo. Após a leitura e análise dos dados de cada artigo, estes foram agrupados em quadros conforme seus resultados. Em seguida foi realizado 
a apresentação e discussão dos resultados em forma narrativa no qual primeiro foi descrito exercício físico, em seguida surdez e logo após as implicações e relação entre ambos.

Figura 1. Fluxograma da seleção de artigo.

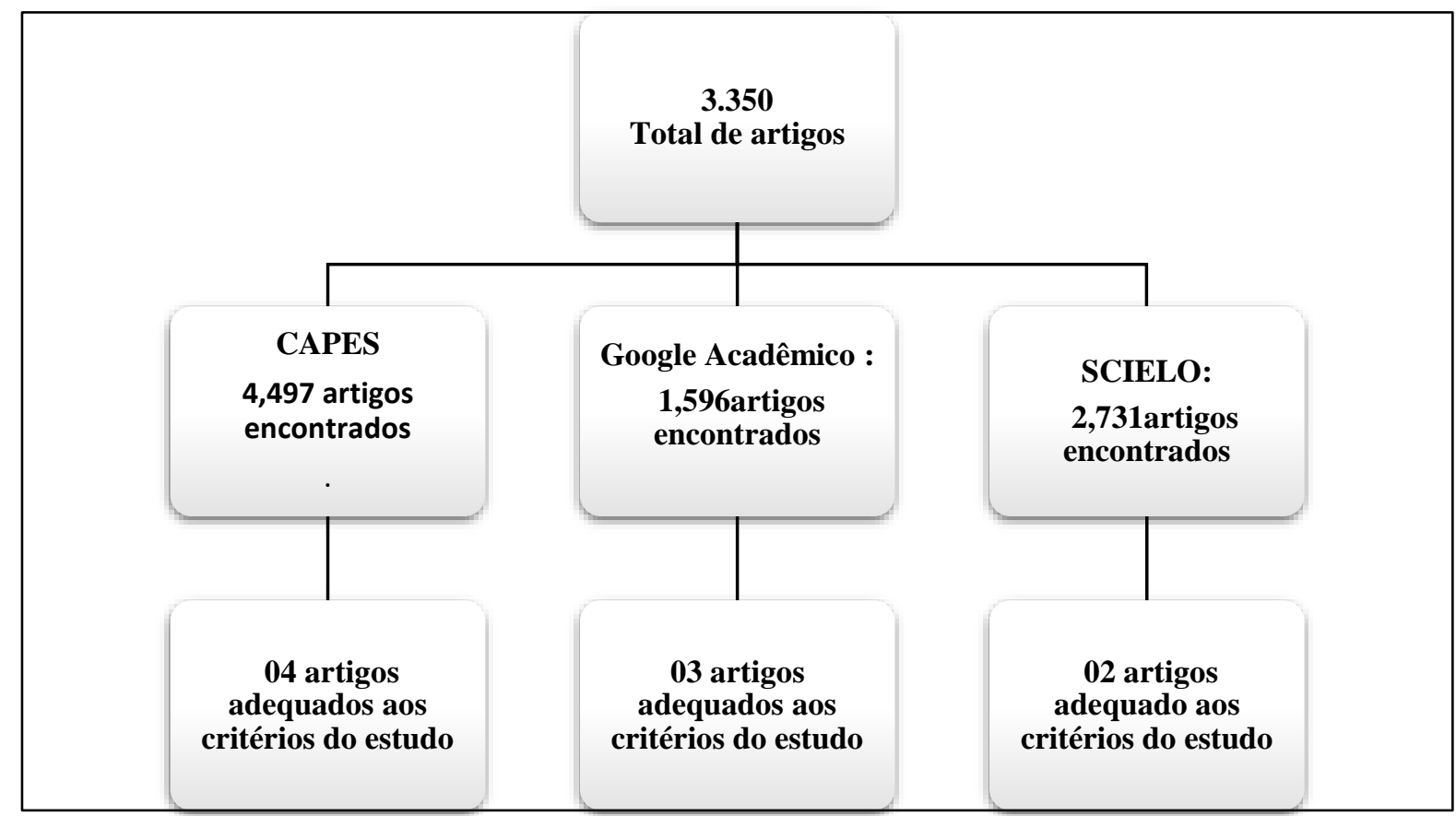

Fonte: Autores.

A atividade física adequada pode promover a saúde e a qualidade de vida de seus praticantes. Além dos benefícios psicossociais, a melhora da aptidão física auxilia significativamente no desempenho das atividades da vida diária, que dependem fortemente do equilíbrio e estão relacionadas às quedas e seus possíveis agravantes, desde a incapacidade de se mover até a dependência de atividades da vida diária. (Bresolin; Silva,2017).

E segundo PolissenI e Ribeiro (2010) a atividade física é reconhecida como fator de proteção para a saúde, sendo seus benefícios associados à redução de doenças crônicas e à diminuição do risco de morte prematura por doenças cardiovasculares. Embora a atividade física seja um importante recurso para políticas de promoção de saúde, o baixo nível e a inatividade física são preocupantes em todo o mundo. Em uma sociedade cada vez mais urbanizada e industrializada, exercícios físicos praticados regularmente são importantes recursos para manter o nível de atividade física recomendado, contudo, é preciso identificar as práticas mais adequadas às necessidades preconizadas como efeito protetor do exercício físico para a saúde

Os surdos podem exercitar sua consciência física e desenvolver suas habilidades motoras por meio da educação física. Do ponto de vista clínico, os surdos não têm restrições ao exercício físico, pois seu desenvolvimento motor é equivalente ao de um indivíduo denominado "normal". (Lima, Yoshioka e Moraes, 2010)

Os efeitos benéficos dos exercícios físicos estão bem descritos na literatura. Porém, em se tratando de escolhas e motivos para a prática de exercícios, é preciso identificar as práticas mais adequadas às necessidades preconizadas como efeito protetor do exercício físico para a saúde (Polisseni; Ribeiro,2010).

De acordo com Abreu e Dias (2017) a atividade física tem efeitos benéficos seja no indivíduo ou na comunidade. Reduz fatores de risco individuais e aumenta a participação do sujeito na sociedade, apresentando-se como uma atividade benéfica para a saúde, acessível, pouco dispendiosa e sem potencial negativo.

Mas, no entanto, sendo o equilíbrio uma habilidade passível de ser desenvolvida e aperfeiçoada por meio de experiências 
corporais, podem ser melhoradas mediante a intervenção por meio de atividades que a promovam. A intervenção com a finalidade de promover o desenvolvimento motor com indivíduos com perda auditiva não requer grandes adaptações e podem ter o objetivo de levá-los a desenvolver a autoconfiança, a melhora da coordenação geral e do equilíbrio, bem como, da velocidade de reação e da agilidade (Bresolin; Silva,2017).

Conceição e Silva (2017) citam que a Educação Física não deve ter como princípio o rendimento esportivo ou a técnica pela técnica, pois essas características trazem a exclusão dos menos habilidosos, ela sempre deve ser pautada pela inclusão de todos. Quando levamos em conta que a Educação Física tem como propósito de estudo a cultura corporal de movimentos, ela se faz de grande valia no desenvolvimento da educação do aluno, por utilizar o esporte, lutas, danças e ginástica ela transfere valores coletivos, sentimentos e comportamentos que levam ao desenvolvimento total de pessoas com deficiências.

Como é caso da deficiência auditiva que é qualquer alteração na percepção normal dos sons e essa alteração pode variar em graus, função da intensidade sonora, medida em decibéis, que o indivíduo é capaz de processar. A perda da habilidade de ouvir pode ser causada por qualquer alteração que fuja da normalidade no processo de audição, seja qual for a causa, tipo e intensidade (Sousa, 2017).

Existem dois tipos de problemas auditivos, o primeiro afeta o ouvido externo ou médio e provoca dificuldades auditivas condutivas, normalmente tratáveis e curáveis. O outro tipo envolve o ouvido interno ou o nervo auditivo e chama-se surdez neurossensorial. A surdez neurossensorial pode se manifestar em qualquer idade, desde o pré-natal até a idade avançada. A cóclea é um órgão muito sensível e vulnerável aos fatores genéticos, às doenças infantis, aos sons muito altos e a alguns medicamentos. Um parto difícil ou prematuro, sobretudo quando o bebê não recebe oxigênio suficiente, pode causar surdez neurossensorial. Ao nascer a criança está sujeita a icterícia, prejudicial ao nervo auditivo, podendo levar a perda de audição (Silva, 2008).

Confrome Speri (2013) na deficiência auditiva leve, a palavra é ouvida, mas certos sons não são percebidos, percebese a fala com voz normal, mas tem dificuldade com voz baixa ou distante, as crianças são consideradas muito distraídas e apresentam dificuldade na comunicação em ambientes ruidosos ou em grupo. Ouvir bem e de forma nítida e discriminadora é essencial para poder receber informações e sequências de aprendizagem escolar. Infelizmente, muitas crianças não conseguem utilizar adequadamente este caminho para aprender porque apresentam déficits parciais ou déficits totais (surdez) de percepção auditiva.

Silva (2008) afirma que a localização de lesão no ouvido, permite a distinguir a surdez: a) de transmissão ou condução; b) de recepção ou percepção; c) mista. A surdez de transmissão decorre de algum tipo de problema no ouvido médio, a lesão existe apenas no aparelho de transmissão: há um impedimento na passagem das vibrações até o ouvido interno. Na surdez de percepção há uma lesão no aparelho de recepção: no órgão de Coti ou nas fibras nervosas condutoras do impulso provocado pelas vibrações sonoras. Está é uma surdez mais complexa porque é o próprio órgão sensorial que pode favorecer as distorções de sensação sonora. No caso da surdez mista tanto o ouvido médio como interno são afetados, e ocorre quando a perda de audição tem componentes condutivos e neurossensorial.

E ainda segundo Jardim, Maciel e Lemos (2017) a deficiência auditiva pode ser também uma perda auditiva congênita que pode ocorrer no período pré-natal, ou nos primeiros dias após o nascimento. As causas mais comuns relacionam-se ao muito baixo peso ao nascimento (abaixo de $1.500 \mathrm{~g}$ ), à ocorrência da hiperbilirrubinemia, às infecções congênitas, como a rubéola, toxoplasmose, citomegalovírus, sífilis e uso de drogas ototóxicas no período neonatal, além das causas de malformação congênita de cabeça e pescoço, ou síndromes.

Silva (2008) classifica os tipos de surdez como o grau e o tipo de perda de audição assim como a idade em que esta ocorreu, vão determinar o tipo de atendimento que o surdo receberá. Enquanto surdo, é considerado o portador de surdez severa e o portador de surdez profunda, que serão descritos a seguir: Portador de Surdez Leve apresenta perda auditiva de até quarenta 
decibéis. Essa perda impede que o aluno perceba igualmente todos os fonemas da palavra. Além disso, a voz fraca ou distante não é ouvida; Porta dor de Surdez Moderada apresenta perda auditiva entre quarenta e setenta decibéis. Esses limites encontramse no nível da percepção da palavra, sendo necessário uma voz de certa intensidade para que seja convenientemente percebida; Portador de Surdez Severa apresenta perda auditiva entre setenta e noventa decibéis. Este tipo de perda permite que ele identifique alguns ruídos familiares e poderá perceber apenas a voz forte, podendo chegar até quatro ou cinco anos sem aprender a falar; Portador de Surdez Profunda apresenta perda auditiva superior a noventa decibéis. A gravidade dessa perda é tal, que o priva das informações auditivas necessárias para perceber e identificar a voz humana, impedindo-o de adquirir naturalmente a linguagem oral.

Os danos que podem ser causados pela perda auditiva de grau leve estão: limitações nas habilidades de prestar atenção, de codificar, compreender, memorizar, manipular e usar efetivamente a informação auditiva, podendo levar até mesmo ao distúrbio do processamento auditivo central. Essa privação auditiva leve na criança, mesmo que temporária, ocasiona atraso na aquisição da fala; déficit de processamento auditivo; distúrbios na integração dos estímulos auditivo-visual (dificultando a leitura); déficit no desenvolvimento cognitivo; problemas de aprendizagem e desempenho escolar; distúrbios articulatórios, tanto a nível de produção, compreensão e expressão verbal (Oliveira, 2016).

A educação física adaptada vem apresentar aspectos positivos, na medida em que os alunos passam a assimilar e a conviver com suas necessidades. Nesse contexto o autor adota a adaptação como "a capacidade da pessoa estar apta a atender as demandas exigidas pela vida". Se considerarmos essas demandas como detalhes de nosso cotidiano, essa adaptação será mais fácil, pode-se dizer que nós nos adaptamos a todo o momento. No entanto é necessário saber se adaptar em cada fase, principalmente quando se trata do ponto de vista emocional. Para o autor, neste contexto, a inclusão está relacionada aos “padrões da normalidade (física, fisiológica, comportamental e social), e que necessitam de superação e compreensão dos ditos 'normais' para serem aceitos". Essa compreensão se dá no momento em que a sociedade transmite seus conhecimentos e passam a ter uma boa relação com os deficientes físicos (Monteiro; Silva, 2010).

No momento que falamos em inclusividade das atividades desportivas, não nos referimos à inclusividade de uma forma que faça a diferença entre o público, nem podemos ignorar que cada um tem as suas particularidades e limitações, mas o que deve ser destacado $\mathrm{O}$ objetivo é reduzir a desigualdade entre públicos. Tolerância desta forma não é por causa da boa audição, mas para que todos os públicos participem de atividades esportivas, porque essa distinção já é uma forma de exclusão. (Alves; Pinto, 2016).

Os conhecimentos acerca da inclusão social bem como os direitos firmados em lei, não devem ser apenas teorias, mas sim posto em prática. Pois assim, estaremos nos adaptando e fazendo assim a verdadeira inclusão desses indivíduos na sociedade, proporcionando alegria, diversão, saúde e qualidade de vida para essas pessoas e sempre seguindo o ritmo da atividade física (Monteiro; Silva,2010).

Gorla (2008) mencionou um método simples e importante em seu trabalho para realizar bem as atividades. Avaliar quem vai realizar a atividade pode não só compreender o indivíduo, mas também desenvolver uma estratégia para a realização da atividade. Abrange alguns tipos de avaliação do desempenho esportivo, como: equilíbrio, agilidade, força, corrida (Alves; Pinto,2016).

Os surdos podem exercitar sua consciência física e desenvolver suas habilidades motoras por meio da educação física. Do ponto de vista clínico, os surdos não têm restrições ao exercício físico, pois seu desenvolvimento motor é equivalente ao de um indivíduo denominado "normal". (Lima; Yoshioka; Moraes, 2010).

O desenvolvimento motor de crianças surdas costuma seguir os padrões de normalidade, não havendo, portanto, nenhuma restrição à prática de atividade física. Quando a surdez é acompanhada de outra deficiência ou de algum outro comprometimento, as possíveis restrições estarão relacionadas a esses(s) outro(s) problema(s) (Figueró, 2015). 
Desse modo, é possível afirmar que o sucesso do desenvolvimento das habilidades motoras7 de alunos surdos está diretamente relacionado a ações interventivas do professor de educação física desde que, estas sejam bem elaboradas e, assim, consequentemente bem desenvolvidas trazendo resultados satisfatórios no que tange ao desenvolvimento do aluno (Conceição; Silva, 2017).

Esporte é o termo utilizado para designar os exercícios físicos realizados individual ou coletivamente que obedecem a regras e não tem fim utilitarista. Os esportes são praticados desde as mais remotas épocas da humanidade, quando, em registros muito antigos, os homens primitivos competiam com a caça e com lutas. Tempos depois, os homens concorriam para adquirir status e poder diante da sociedade, ou ainda para produzir fronteiras culturais como no caso do Futebol Americano, criado para produzir uma "americanização" da cultura inglesa e, então, fazer o caminho inverso da colonização e implantar-los comercialmente na Inglaterra a partir dos Estados Unidos (Franco; Paludo; Lebedeff, 2015).

O esporte pode ser um elo fundamental entre os surdos, a fim de adquirir sua própria língua no contato com os seus semelhantes, pois esta participação vai além de desenvolver-se fisicamente, oportunizando a comunicação e uma gama de informações fundamentais para a consolidação de sua identidade como surdo (Di Franco, 2014).

Quando falamos de inclusão de atividades físicas, não queremos nos referir a uma inclusão de modo que crie uma diferenciação entre o público, não sendo também omisso que cada um tem suas especificidades e suas limitações, mas o que deve sobressair é o propósito de diminuir as desigualdades entre os públicos, de uma maneira que a inclusão não seja pelo fato de ter ou não uma audição favorável, mas sim, de levar a atividade física para todos os públicos, pois fazer essa diferenciação já seria uma forma de exclusão (Alves; Pinto, 2016).

Os surdos podem praticar qualquer tipo de esporte e de atividade rítmica. No caso dos esportes, não há necessidade de qualquer adaptação na forma de ensinar, conduzir ou arbitrar. Tampouco há adaptações nas regras de cada modalidade. Já as atividades rítmicas, se envolverem coreografia, costumam demandar um pouco mais de tempo de treinamento, devido à necessidade de internalizar o tempo e o andamento da execução dos movimentos sem o auxílio de uma trilha sonora (mesmo com boa amplificação os surdos não conseguem perceber a maior parte das nuances de uma música (Texeira,2009).

Por meio de um estudo para se avaliar o nível de coordenação motora em crianças surdas e a influência das aulas de Educação Física nas mesmas, utilizando a bateria de testes KTK e intervenções de iniciação ao basquetebol, foi possível comprovar, por meio do resultado de uma pesquisa que um programa de atividades físicas bem orientadas são fundamentais para aquisição de habilidades motoras em crianças com deficiência auditiva (Conceição; Silva, 2017).

\section{Conclusão}

Diante das abordagens acima citado pôde-se constatar que as dificuldades encontradas pelos deficientes auditivos em praticarem atividade física sempre existiram, mas os mesmos sempre lutaram pelos seus direitos até os dias atuais, e sempre em busca de melhorias e qualidade de vida. Sua trajetória foi marcada, em nossa história, pelas dificuldades encontradas tanto pelos surdos como pelos profissionais de educação física que tiveram que buscar meios para adaptar os exercícios físicos para que os surdos pudessem interagir com o restante da população.

Diante das abordagens acima citado pôde-se constatar que as dificuldades encontradas pelos deficientes auditivos em praticarem atividade física sempre existiram, mas os mesmos sempre lutaram pelos seus direitos até os dias atuais, e sempre em busca de melhorias e qualidade de vida. Sua trajetória foi marcada, em nossa história, pelas dificuldades encontradas tanto pelos surdos como pelos profissionais de educação física que tiveram que buscar meios para adaptar os exercícios físicos para que os surdos pudessem interagir com o restante da população.

Verificou-se a importância em se adaptar os exercícios para facilitar a inclusão social dos deficientes seja ele físico, mental, auditivo ou visual. Mas para que ocorra a verdadeira inclusão social faz se necessário uma adaptação não apenas nos 
exercícios como também nas instituições de ensino, nas áreas de lazer como: praças, clubes, etc.; em repartições públicas, meios de transportes, etc., porém, ainda, há muitas barreiras às quais os deficientes físicos deverão percorrer em relação a esse processo de inclusão. Pois sabemos que apesar dos deficientes terem alcançados muitas, conquista no que diz respeito a sua inclusão nas atividades físicas, sabemos que ainda há muito a se fazer para que os deficientes se sintam realizados e possam usufruir de seus direitos como todo e qualquer cidadão.

Acredita-se que a inclusão dos surdos nas atividades físicas só será possível no momento em que haja uma conscientização da sociedade da importância dos exercícios físicos, para os deficientes auditivos, a usufruírem seus direitos e dos prazeres que a vida possa lhe oferecer. E isso seria fácil se houvesse adaptação em todos os espaços públicos, e fossem oferecidas atividades físicas para toda sociedade ensinando desde a criança até o adulto a movimentar-se melhor, dominando assim seus movimentos seja ele um simples levantar de braço ou um giro de cento e oitenta graus. Assim nossa sociedade seria mais humilde e justa com toda a população, independentemente de sua cor, raça ou etnia.

\section{Referências}

Abreu, M. O., \& Dias, I. S. (2017). Exercício físico, saúde mental e qualidade de vida na ESECS/IPL. Psicologia, Saúde e Doenças, $18(2), 512-526$.

Akınoğlu, B., \& Kocahan, T. (2019). Stabilization training versus equilibrium training in karate athletes with deafness. Journal of exercise rehabilitation, 15(4), 576.

Alves, L. K. C., \& Pinto, F. R. M. (2016). O Surdo e a Prática de Atividades Físicas Mediado por um Educador Físico. Afluente: Revista de Letras e Linguística, 98-115.

Boaventura, R. D. S., Castelli, M. D. S., \& Barata, T. C. (2012). Os benefícios da atividade física para a pessoa com deficiência. Revista OMNIA Saúde, 6(1), 51-61.

Bresolin, D. S., \& da Silva, M. A. (2018). Equilíbrio comparado por avaliação do cop entre surdos praticantes de exercício físico e não praticantes. Do Corpo: ciências e artes, 7(1).

Conceição, R. M., \& dos Reis Viana, J. B. (2017). O desenvolvimento motor do aluno surdo na educação física. acta Brasileira do Movimento Humano, 7(3), $1-9$.

Di Franco, M. A. R. (2014). Esportes surdos na constituição do ser social: o resgate histórico sob a perspectiva da educação ambiental (Master's thesis).

Di Franco, M. A. R., Paludo, S. D. S., \& Lebedeff, T. B. (2015). Esportes surdos na constituição do ser social: uma compreensão histórica sob a perspectiva da Educação Ambiental. Revista Educação Especial, 28(52), 365-376.

Figueró, L. K. (2015). Uma comparação do desenvolvimento motor entre crianças surdas e ouvintes da educação infantil de uma escola privada de Sapiranga.

Jardim, D. S., Maciel, F. J., \& Lemos, S. M. A. (2017). Perda auditiva incapacitante: análise de fatores associados. Audiology-Communication Research, 22.

Lima, C. M. D. (2019). Pessoas surdas e as barreiras atitudinais encontradas para a prática de atividade física (Bachelor's thesis).

Lima, T. C. S; Yoshioka, M. C.da C. P; MORAES, R.de. Avaliação do desenvolvimento motor de crianças surdas.2010

Monteiro, M. S. (2006). História dos movimentos dos surdos e o reconhecimento da Libras no Brasil. ETD-Educação Temática Digital, 7(2), $292-305$.

Monteiro, J., \& Silva, M. (2010). DA. importância da atividade física para os deficientes físicos. EFDeportes. com. Revista Digital, 15(148), 1-1.

Oliveira, Patrícia Maria de. Estimulação Cognitiva.2016.

Polisseni, M. L. D. C., \& Ribeiro, L. C. (2014). Exercício físico como fator de proteção para a saúde em servidores públicos. Revista Brasileira de Medicina do Esporte, 20(5), 340-344.

Santos, K. R. O. R. P. (2011). Formação continuada e necessidades formativas de professores na educação de surdos da rede pública da cidade do Rio de Janeiro (Doctoral dissertation, Tese de Doutorado). Universidade Metodista de Piracicaba, São Paulo, Brasil).

dos Santos, E. C. G. (2012, August). Surdez e Atividade Física. In III Congresso Norte-brasileiro de Ciências do Esporte.

Sheffield, A. M., \& Smith, R. J. (2019). The epidemiology of deafness. Cold Spring Harbor perspectives in medicine, 9(9), a033258.

Silva, D. P., de Freitas Silva, P. R. P., de Souza, J. V., \& Cotes, M. (2019). Atividade física de aventura na natureza para pessoas com deficiência. LICERERevista do Programa de Pós-graduação Interdisciplinar em Estudos do Lazer, 22(2), 268-296.

Speri, M. R. B. (2013). A criança com deficiência auditiva: da suspeita ao processo de reabilitação fonoaudiológica. Verba Volant, 4(01), 40-64.

Speri, M. R. B. (2013). A criança com deficiência auditiva: da suspeita ao processo de reabilitação fonoaudiológica. Verba Volant, 4(01), 40-64. 
Research, Society and Development, v. 10, n. 9, e0910917723, 2021

(CC BY 4.0) | ISSN 2525-3409 | DOI: http://dx.doi.org/10.33448/rsd-v10i9.17723

Texeira, L.Atividades Fisicas para deficientes auditivos. São Paulo.2009.

Tzanetakos, N., Papastergiou, M., Vernadakis, N., \& Antoniou, P. (2017). Utilizing physically interactive videogames for the balance training of adolescents with deafness within a physical education course. Journal of Physical Education and Sport, 17(2), 614. 